\title{
ON THE CONSTRUCTION OF SPARSE TENSOR PRODUCT SPACES
}

\author{
MICHAEL GRIEBEL AND HELMUT HARBRECHT
}

\begin{abstract}
Let $\Omega_{1} \subset \mathbb{R}^{n_{1}}$ and $\Omega_{2} \subset \mathbb{R}^{n_{2}}$ be two given domains and consider on each domain a multiscale sequence of ansatz spaces of polynomial exactness $r_{1}$ and $r_{2}$, respectively. In this paper, we study the optimal construction of sparse tensor products made from these spaces. In particular, we derive the resulting cost complexities to approximate functions with anisotropic and isotropic smoothness on the tensor product domain $\Omega_{1} \times \Omega_{2}$. Numerical results validate our theoretical findings.
\end{abstract}

\section{INTRODUCTION}

Many problems in science and engineering lead to problems which are defined on the tensor product of two domains $\Omega_{1} \subset \mathbb{R}^{n_{1}}$ and $\Omega_{2} \subset \mathbb{R}^{n_{2}}$. Examples arise from the second moment analysis of partial differential domains with stochastic input parameters [12, 13, 22, 20], two-scale homogenization [1, 4, 14, radiosity models and radiative transfer [23, or space-time discretizations of parabolic problems [9, 19].

A straightforward discretization uses tensor products of all basis functions from suitable finite dimensional ansatz spaces $V_{J}^{(1)}$ and $V_{J}^{(2)}$ which are defined on each domain separately. This leads to the full tensor product space $V_{J}^{(1)} \otimes V_{J}^{(2)}$. However, in general, the full tensor product space contains too many degrees of freedom such that desirable realistic simulations are still beyond current computing capacities. For this reason, the efficient discretization of functions on product domains is an important task in numerical analysis and scientific computing.

In the present paper, we focus on the construction of sparse tensor product spaces, also known as sparse grids 2, 24. Starting points are multilevel decompositions of the ansatz spaces

$$
V_{J}^{(i)}=W_{0}^{(i)} \oplus W_{1}^{(i)} \oplus \cdots \oplus W_{J}^{(i)}, \quad i=1,2,
$$

which can be constructed via hierarchical bases, interpolets or wavelet-like bases. From this, the regular sparse tensor product space is defined according to

$$
\widehat{V}_{J}^{r e g}=\bigoplus_{j_{1}+j_{2} \leq J} W_{j_{1}}^{(1)} \otimes W_{j_{2}}^{(2)} ;
$$

see e.g. [2, 8, 10, 24]. Its approximation power is nearly as good as that of the corresponding full tensor product space if the functions to be approximated provide additional smoothness in terms of bounded mixed derivatives. The regular sparse grid is optimal with respect to the $L^{2}$-norm if both domains have the same dimension and are equipped with the same type of functions.

Received by the editor May 27, 2011 and, in revised form, September 26, 2011 and October 15,2011

2010 Mathematics Subject Classification. Primary 41A17, 41A25, 41A30, 41A65. 
Nevertheless, to the best of our knowledge, it has not been systematically studied in the literature what the most efficient construction of sparse tensor product spaces is if the spatial dimension of the underlying domains or the polynomial exactness (and thus the approximation power) of the ansatz spaces differ. Here, the following questions arise: Should the degrees of freedom or the approximation power of the univariate ansatz spaces be equilibrated? Or is it preferable to construct the sparse tensor product space such that an equilibrated cost-benefit rate (see [2]) is guaranteed?

In the present paper, we will answer these questions for the case that the approximation error is measured in the $L^{2}\left(\Omega_{1} \times \Omega_{2}\right)$-norm. Then, the sparse tensor product spaces contain all products $W_{j_{1}}^{(1)} \otimes W_{j_{2}}^{(2)}$ with indices $\left(j_{1}, j_{2}\right)$ contained inside a specific triangular subset of the $j_{1}-j_{2}$ plane with base dependent on $n_{1}, n_{2}, r_{1}, r_{2}$. Here, besides the approximation error of functions from Sobolev spaces of dominating mixed smoothness, we also study the approximation error of functions from isotropic Sobolev spaces.

In the case of smooth functions, there is a whole range of sparse tensor product spaces which possess the same optimal convergence rate. However, in the case of functions with limited regular or mixed Sobolev smoothness, it turns out that the sparse tensor product space, which equilibrates the number of degrees of freedom (see Section 3), is superior to all the other sparse tensor product spaces under consideration.

To keep the discussion simple, we restrict ourselves to two-fold tensor product domains $\Omega_{1} \times \Omega_{2}$ in this paper. In practice, also problems on arbitrary tensor product domains $\Omega_{1} \times \Omega_{2} \times \cdots \times \Omega_{m}$ may appear; see e.g. [2, 11, 16, 17, 18, 21, 22]. We believe that our results can be generalized to such $m$-fold tensor product domains which, however, is rather technical and not straightforward. This will therefore need future research (see also the concluding remarks in Section 8).

The remainder of this paper is organized as follows. In Section 2, we specify the requirements of the multiscale hierarchies on each subdomain. Then, in Section 3 , we construct general sparse tensor product spaces. In Section 4 we study their properties. Section 5 is dedicated to the comparison of the cost complexities for the approximation of functions with anisotropic and isotropic smoothness. In Section 6 , we provide the results of our numerical experiments. They are in good agreement with the presented theory. Finally, in Section [7, we carry over our results from the $L^{2}\left(\Omega_{1} \times \Omega_{2}\right)$-error estimate to the more general $H_{\text {mix }}^{q_{1}, q_{2}}\left(\Omega_{1} \times \Omega_{2}\right)$-error estimate.

Throughout this paper, the notion "essential" in the context of complexity estimates means "up to logarithmic terms". Moreover, to avoid the repeated use of generic but unspecified constants, we denote by $C \lesssim D$ that $C$ is bounded by a multiple of $D$ independently of parameters which $C$ and $D$ may depend on. Obviously, $C \gtrsim D$ is defined as $D \lesssim C$, and $C \sim D$ as $C \lesssim D$ and $C \gtrsim D$.

\section{Approximation on the subdomains}

Let $\Omega \subset \mathbb{R}^{n}$ be a sufficiently smooth, bounded domain. We consider a nested sequence of finite dimensional subspaces

$$
V_{0} \subset V_{1} \subset \cdots \subset V_{j} \subset \cdots \subset L^{2}(\Omega),
$$


which consists of piecewise polynomial ansatz functions $V_{j}=\operatorname{span}\left\{\varphi_{j, k}: k \in \Delta_{j}\right\}$ $\left(\Delta_{j}\right.$ denotes a suitable index set), such that $\operatorname{dim} V_{j} \sim 2^{j n}$ and

$$
L^{2}(\Omega)=\overline{\bigcup_{j \in \mathbb{N}_{0}} V_{j}} .
$$

Since we will use the spaces $V_{j}$ for the approximation of functions, we assume that the approximation property

$$
\inf _{v_{j} \in V_{j}}\left\|u-v_{j}\right\|_{L^{2}(\Omega)} \lesssim h_{j}^{s}\|u\|_{H^{s}(\Omega)}, \quad u \in H^{s}(\Omega),
$$

holds for $0 \leq s \leq r$ uniformly in $j$. Here we set $h_{j}:=2^{-j}$, i.e., $h_{j}$ corresponds to the width of the mesh associated with the subspace $V_{j}$ on $\Omega$. Note that the integer $r$ refers to the polynomial exactness, that is, the maximal order of polynomials which are locally contained in the space $V_{j}$.

We now introduce a wavelet basis associated with the multiscale analysis (2.1) and (2.2) as follows. The wavelets $\Psi_{j}:=\left\{\psi_{j, k}: k \in \nabla_{j}\right\}$, where $\nabla_{j}:=\Delta_{j} \backslash \Delta_{j-1}$, are the bases of the complementary spaces $W_{j}$ of $V_{j-1}$ in $V_{j}$, i.e.,

$$
V_{j}=V_{j-1} \oplus W_{j}, \quad V_{j-1} \cap W_{j}=\{0\}, \quad W_{j}=\operatorname{span}\left\{\Psi_{j}\right\} .
$$

Recursively, we obtain

$$
V_{J}=\bigoplus_{j=0}^{J} W_{j}, \quad W_{0}:=V_{0},
$$

and thus, with

$$
\Psi_{J}:=\bigcup_{j=0}^{J} \Psi_{j}, \quad \Psi_{0}:=\Phi_{0},
$$

we get a wavelet basis in $V_{J}$. A final requirement is that the infinite collection $\Psi:=\bigcup_{j>0} \Psi_{j}$ forms a Riesz basis of $L^{2}(\Omega)$. Then, there exists also a biorthogonal, or dual, wavelet basis $\widetilde{\Psi}=\bigcup_{j>0} \widetilde{\Psi}_{j}=\left\{\widetilde{\psi}_{j, k}: k \in \nabla_{j}, j \geq 0\right\}$ which defines a dual multiscale analysis; see, e.g., [5] for further details. In particular, each function $f \in L^{2}(\Omega)$ admits the unique representation

$$
f=\sum_{j=0}^{\infty} \sum_{k \in \nabla_{j}}\left(f, \widetilde{\psi}_{j, k}\right)_{L^{2}(\Omega)} \psi_{j, k} .
$$

With the definition of the projections

$$
Q_{j}: L^{2}(\Omega) \rightarrow W_{j}, \quad Q_{j} f=\sum_{k \in \nabla_{j}}\left(f, \widetilde{\psi}_{j, k}\right)_{L^{2}(\Omega)} \psi_{j, k},
$$

the atomic decomposition (2.4) gives rise to the multilevel decomposition

$$
f=\sum_{j=0}^{\infty} Q_{j} f .
$$

In particular, for any $f \in H^{s}(\Omega)$, the approximation property (2.3) induces the estimate

$$
\left\|Q_{j} f\right\|_{L^{2}(\Omega)} \lesssim 2^{-j s}\|f\|_{H^{s}(\Omega)}, \quad 0 \leq s \leq r .
$$




\section{Sparse tensor PRoduct Spaces}

Consider now two domains $\Omega_{1} \subset \mathbb{R}^{n_{1}}$ and $\Omega_{2} \subset \mathbb{R}^{n_{2}}$ with $n_{1}, n_{2} \in \mathbb{N}$. We aim at the approximation of functions in $L^{2}\left(\Omega_{1} \times \Omega_{2}\right)$. To this end, we assume individually for each subdomain $\Omega_{i}, i=1,2$, the multiscale analyses

$$
V_{0}^{(i)} \subset V_{1}^{(i)} \subset V_{2}^{(i)} \subset \cdots \subset L^{2}\left(\Omega_{i}\right), \quad V_{0}^{(i)}=\operatorname{span}\left\{\Phi_{j}^{(i)}\right\}, \quad i=1,2,
$$

with associated complementary spaces

$$
V_{j}^{(i)}=V_{j-1}^{(i)} \oplus W_{j}^{(i)}, \quad V_{j-1}^{(i)} \cap W_{j}^{(i)}=\{0\}, \quad W_{j}^{(i)}=\operatorname{span}\left\{\Psi_{j}^{(i)}\right\} .
$$

Furthermore, let us denote the polynomial exactnesses of the spaces $V_{j}^{(1)}$ and $V_{j}^{(2)}$ by $r_{1}$ and $r_{2}$, respectively.

In this paper, we study the special sparse tensor product space 1

$$
\widehat{V}_{J}^{\sigma}:=\bigoplus_{j_{1} \sigma+\frac{j_{2}}{\sigma} \leq J} W_{j_{1}}^{(1)} \otimes W_{j_{2}}^{(2)}
$$

for an arbitrary parameter $\sigma>0$. In particular, the index pairs $\left(j_{1}, j_{2}\right) \in \mathbb{N}_{0} \times \mathbb{N}_{0}$ of the included tensor product spaces $W_{j_{1}}^{(1)} \otimes W_{j_{2}}^{(2)}$ satisfy the relations

$$
0 \leq j_{1} \leq \frac{1}{\sigma} J-\frac{1}{\sigma^{2}} j_{2}, \quad 0 \leq j_{2} \leq \sigma J-\sigma^{2} j_{1} .
$$

Note that this ansatz would not be optimal if we were interested in approximation errors with respect to norms other than the $L^{2}\left(\Omega_{1} \times \Omega_{2}\right)$-norm. We would then no longer obtain spaces with triangular index set, but so-called generalized sparse grid spaces [2, 6, 7, 8] with more complicatedly shaped index sets which no longer exhibit a linear relation between $j_{1}$ and $j_{2}$. For example, the choice of the $H^{1}\left(\Omega_{1} \times \Omega_{2}\right)$ seminorm would lead to the so-called energy based sparse grids; see [2]. Results for other norms and smoothness classes can be found in [8].

Here, we restrict ourselves to the $L^{2}\left(\Omega_{1} \times \Omega_{2}\right)$-norm but consider $\Omega_{1}$ and $\Omega_{2}$ with $n_{1}$ and $n_{2}$ in general not equal and possibly equipped with different polynomial ansatz spaces of exactness $r_{1}$ and $r_{2}$, respectively.

Reasonable choices of the parameter $\sigma$ could then be as follows.

- We may equilibrate the degrees of freedom in all tensor product spaces $W_{j_{1}}^{(1)} \otimes W_{j_{2}}^{(2)}$ whose indices $\left(j_{1}, j_{2}\right)$ satisfy $j_{1} \sigma+j_{2} / \sigma=J$. This choice leads to $\sigma=\sqrt{n_{1} / n_{2}}$.

- The sparse tensor product space $\widehat{V}_{J}^{\sigma}$ (3.6) can be rewritten as

$$
\widehat{V}_{J}^{\sigma}=\sum_{j_{1} \sigma+j_{2} / \sigma=J} V_{j_{1}}^{(1)} \otimes V_{j_{2}}^{(2)} .
$$

Then, it can be seen easily that the choice $\sigma:=\sqrt{r_{1} / r_{2}}$ equilibrates the approximation power of the contained tensor product spaces $V_{j_{1}}^{(1)} \otimes V_{j_{2}}^{(2)}$.

- Following the idea of an equilibrated cost-benefit rate (see [2]), we get the condition

$$
2^{j_{1}\left(n_{1}+r_{1}\right)} \cdot 2^{j_{2}\left(n_{2}+r_{2}\right)} \stackrel{!}{=} 2^{J \cdot c o n s t} .
$$

Then, by choosing as const $=\sqrt{\left(n_{1}+r_{1}\right)\left(n_{2}+r_{2}\right)}$ we arrive at $\sigma=\sqrt{\frac{n_{1}+r_{1}}{n_{2}+r_{2}}}$.

\footnotetext{
${ }^{1}$ Here and in the following, the summation limits are in general not natural numbers and must of course be rounded properly. We leave this to the reader to avoid cumbersome floor/ceilnotations.
} 


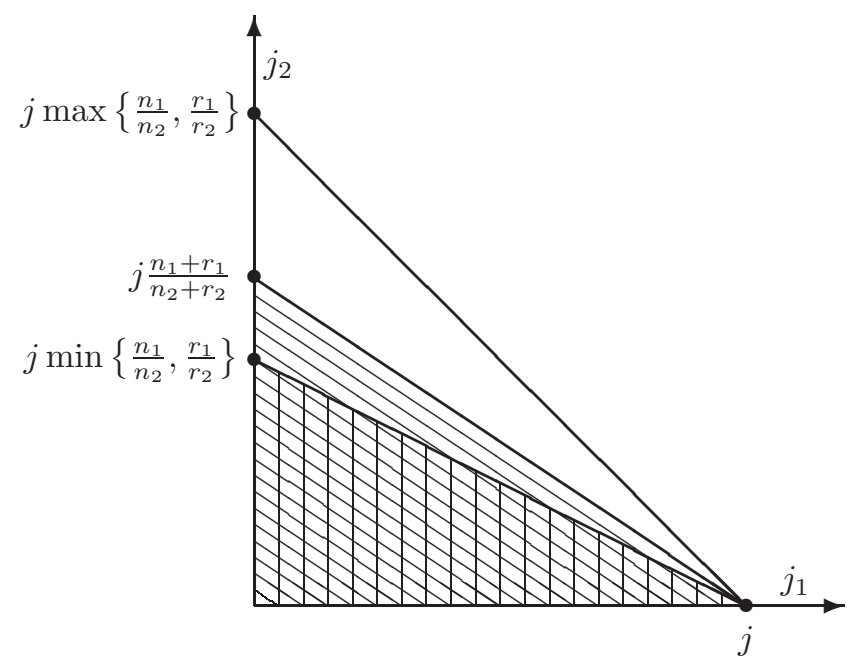

Figure 3.1. The index sets $\left(j_{1}, j_{2}\right)$ of the spaces $\widehat{V}_{J}^{\sigma}$ with the same intersection point $j$ on the $j_{1}$-axis for the three particular choices of $\sigma$.

In Figure 3.1, we display the index sets $\left(j_{1}, j_{2}\right)$ which belong to the associated sparse tensor product spaces $\widehat{V}_{J}^{\sigma}$ for these three cases of $\sigma$.

\section{Properties of the Sparse tensor ProduCt SPACES}

In the following section, we present results for the approximation of functions in the sparse tensor product spaces $\widehat{V}_{J}^{\sigma}$ with arbitrary $\sigma>0$. First, we count the degrees of freedom which are contained in these spaces.

Theorem 4.1. The dimension of the sparse tensor product space

$$
\widehat{V}_{J}^{\sigma}=\sum_{\sigma j_{1}+j_{2} / \sigma \leq J} W_{j_{1}}^{(1)} \otimes W_{j_{2}}^{(2)}
$$

is essentially $\mathcal{O}\left(2^{J \max \left\{n_{1} / \sigma, n_{2} \sigma\right\}}\right)$. More precisely, it holds that

$$
\operatorname{dim} \widehat{V}_{J}^{\sigma} \lesssim \begin{cases}2^{J \max \left\{n_{1} / \sigma, n_{2} \sigma\right\}}, & \text { if } n_{1} / \sigma \neq n_{2} \sigma \\ 2^{J n_{2} \sigma} J, & \text { if } n_{1} / \sigma=n_{2} \sigma .\end{cases}
$$

Proof. Since $\operatorname{dim}\left(W_{j_{1}}^{(1)} \otimes W_{j_{2}}^{(2)}\right) \sim 2^{j_{1} n_{1}+j_{2} n_{2}}$, it holds that

$$
\operatorname{dim} \widehat{V}_{J}^{\sigma} \sim \sum_{j_{1}=0}^{J / \sigma} \sum_{j_{2}=0}^{\sigma J-\sigma^{2} j_{1}} 2^{j_{1} n_{1}+j_{2} n_{2}} \lesssim 2^{J n_{2} \sigma} \sum_{j_{1}=0}^{J / \sigma} 2^{j_{1}\left(n_{1}-n_{2} \sigma^{2}\right)} .
$$

Now, if $n_{1} / \sigma<n_{2} \sigma$, the exponent in the sum is always negative. This implies

$$
\operatorname{dim} \widehat{V}_{J}^{\sigma} \lesssim 2^{J n_{2} \sigma}
$$

If $n_{1} / \sigma>n_{2} \sigma$, the exponent is always positive and it follows that

$$
\operatorname{dim} \widehat{V}_{J}^{\sigma} \lesssim 2^{J n_{2} \sigma} 2^{J / \sigma\left(n_{1}-n_{2} \sigma^{2}\right)}=2^{J n_{1} / \sigma} .
$$


In the case of $n_{1} / \sigma=n_{2} \sigma$ we obtain

$$
\operatorname{dim} \widehat{V}_{J}^{\sigma} \lesssim 2^{J n_{2} \sigma} \sum_{j_{1}=0}^{J / \sigma} 1 \lesssim 2^{J n_{2} \sigma} \frac{J}{\sigma}
$$

The combination of (4.8)-(4.10) yields the desired result.

Remark 4.2. (i) Note that the constant in estimate (4.7) depends on the particular choice of $\sigma$.

(ii) Estimate (4.7) is sharp since it holds $\operatorname{dim} W_{J / \sigma}^{(1)} \sim 2^{J n_{1} / \sigma}$ whereas $\operatorname{dim} W_{\sigma J}^{(2)} \sim$ $2^{J n_{2} \sigma}$. Thus, we also have the lower bound $2^{J \max \left\{n_{1} / \sigma, n_{2} \sigma\right\}}$.

(iii) The full tensor product space $V_{J / \sigma}^{(1)} \otimes V_{J \sigma}^{(2)}$ consists of $2^{J\left(n_{1} / \sigma+n_{2} \sigma\right)}$ degrees of freedom.

In view of just optimal cost we would get $n_{1} / \sigma=n_{2} \sigma$, that is, $\sigma=\sqrt{n_{1} / n_{2}}$, from balancing the terms in $\max \left\{n_{1} / \sigma, n_{2} \sigma\right\}$. But this choice of $\widehat{V}_{J}^{\sigma}$ need not give us necessarily the best rate of approximation yet. Therefore, our next result is concerned with the rate of approximation in the sparse tensor spaces $\widehat{V}_{J}^{\sigma}$. To this end, for $s_{1}, s_{2} \geq 0$, we introduce the anisotropic Sobolev spaces

$$
H_{\text {mix }}^{s_{1}, s_{2}}\left(\Omega_{1} \times \Omega_{2}\right):=H^{s_{1}}\left(\Omega_{1}\right) \otimes H^{s_{2}}\left(\Omega_{2}\right) .
$$

Obviously, the highest possible rate of convergence is attained in the space $H_{\text {mix }}^{r_{1}, r_{2}}\left(\Omega_{1} \times \Omega_{2}\right)$. Therefore, in the following theorem, we restrict ourselves, without loss of generality, to $s_{1} \leq r_{1}$ and $s_{2} \leq r_{2}$.

Theorem 4.3. Let $0<s_{1} \leq r_{1}, 0<s_{2} \leq r_{2}$ and $f \in H_{m i x}^{s_{1}, s_{2}}\left(\Omega_{1} \times \Omega_{2}\right)$. Then, the approximation

$$
\widehat{f}_{J}=\sum_{j_{1} \sigma+\frac{j_{2}}{\sigma} \leq J}\left(Q_{j_{1}}^{(1)} \otimes Q_{j_{2}}^{(2)}\right) f \in \widehat{V}_{J}^{\sigma}
$$

satisfies

$$
\left\|f-\widehat{f}_{J}\right\|_{L^{2}\left(\Omega_{1} \times \Omega_{2}\right)} \lesssim \begin{cases}2^{-J \min \left\{s_{1} / \sigma, s_{2} \sigma\right\}}\|f\|_{H_{\text {mix }}^{s_{1}, s_{2}}\left(\Omega_{1} \times \Omega_{2}\right)}, & \text { if } s_{1} / \sigma \neq s_{2} \sigma, \\ 2^{-J s_{1} / \sigma} \sqrt{J}\|f\|_{H_{\text {mix }}^{s_{1}, s_{2}}\left(\Omega_{1} \times \Omega_{2}\right)}, & \text { if } s_{1} / \sigma=s_{2} \sigma .\end{cases}
$$

Proof. It follows by standard tensor product arguments from (2.5) that

$$
\begin{aligned}
\left\|f-\widehat{f}_{J}\right\|_{L^{2}\left(\Omega_{1} \times \Omega_{2}\right)}^{2} & \lesssim \sum_{j_{1} \sigma+\frac{j_{2}}{\sigma}>J}\left\|\left(Q_{j_{1}}^{(1)} \otimes Q_{j_{2}}^{(2)}\right) f\right\|_{L^{2}\left(\Omega_{1} \times \Omega_{2}\right)}^{2} \\
& \lesssim \sum_{j_{1} \sigma+\frac{j_{2}}{\sigma}>J} 2^{-2\left(s_{1} j_{1}+s_{2} j_{2}\right)}\|f\|_{H_{m i x}^{s_{1}, s_{2}}\left(\Omega_{1} \times \Omega_{2}\right)}^{2} .
\end{aligned}
$$

Now, we split the index set

$$
I:=\left\{\left(j_{1}, j_{2}\right): j_{1} \sigma+\frac{j_{2}}{\sigma}>J\right\}
$$

into two disjoint sets $I=I_{1} \cup I_{2}$ given by

$$
\begin{aligned}
& I_{1}:=\left\{\left(j_{1}, j_{2}\right): 0 \leq j_{1} \leq \frac{J}{\sigma}, J \sigma-j_{1} \sigma^{2}<j_{2}\right\}, \\
& I_{2}:=\left\{\left(j_{1}, j_{2}\right): \frac{J}{\sigma}<j_{1}, 0 \leq j_{2}\right\} .
\end{aligned}
$$


Thus, we get

$$
\begin{aligned}
\| f & -\widehat{f}_{J} \|_{L^{2}\left(\Omega_{1} \times \Omega_{2}\right)}^{2} \sum^{-2\left(s_{1} j_{1}+s_{2} j_{2}\right)} \\
& \lesssim\left\{\sum_{j_{1}=0}^{J / \sigma} \sum_{j_{2}=J \sigma-j_{1} \sigma^{2}+1}^{\infty}+\sum_{j_{1}=J / \sigma+1}^{\infty} \sum_{j_{2}=0}^{\infty} 2^{-2\left(s_{1} j_{1}+s_{2} j_{2}\right)}\right\}\|f\|_{H_{m i x}^{s_{1}, s_{2}}\left(\Omega_{1} \times \Omega_{2}\right)}^{2} \\
& \lesssim\left\{\sum_{j_{1}=0}^{J / \sigma} 2^{-2 j_{1}\left(s_{1}-s_{2} \sigma^{2}\right)-2 J s_{2} \sigma}+\sum_{j_{1}=J / \sigma+1}^{\infty} 2^{-2 s_{1} j_{1}}\right\}\|f\|_{H_{m i x}^{s_{1}, s_{2}}\left(\Omega_{1} \times \Omega_{2}\right)}^{2} \\
& \lesssim\left\{\sum_{j_{1}=0}^{J / \sigma} 2^{-2 j_{1}\left(s_{1}-s_{2} \sigma^{2}\right)-2 J s_{2} \sigma}+2^{-2 J \frac{s_{1}}{\sigma}}\right\}\|f\|_{H_{m i x}^{s_{1}, s_{2}}\left(\Omega_{1} \times \Omega_{2}\right)}^{2} \\
& =2^{-2 J s_{2} \sigma}\left\{\sum_{j_{1}=0}^{J / \sigma} 2^{-2 j_{1} \sigma\left(\frac{s_{1}}{\sigma}-s_{2} \sigma\right)}+2^{-2 J\left(\frac{s_{1}}{\sigma}-s_{2} \sigma\right)}\right\}\|f\|_{H_{m i x}^{s_{1}, s_{2}}\left(\Omega_{1} \times \Omega_{2}\right)}^{2} .
\end{aligned}
$$

To obtain an estimate for this expression, we again distinguish three cases.

In the case $s_{1} / \sigma<s_{2} \sigma$, we find

$$
\begin{aligned}
\left\|f-\widehat{f}_{J}\right\|_{L^{2}\left(\Omega_{1} \times \Omega_{2}\right)}^{2} & \lesssim 2^{-2 J s_{2} \sigma}\left\{2^{-2 J\left(\frac{s_{1}}{\sigma}-s_{2} \sigma\right)}+2^{-2 J\left(\frac{s_{1}}{\sigma}-s_{2} \sigma\right)}\right\}\|f\|_{H_{m i x}^{s_{1}, s_{2}}\left(\Omega_{1} \times \Omega_{2}\right)}^{2} \\
& \lesssim 2^{-2 J \frac{s_{1}}{\sigma}}\|f\|_{H_{m i x}^{s_{1}, s_{2}}\left(\Omega_{1} \times \Omega_{2}\right)}^{2} .
\end{aligned}
$$

If $s_{1} / \sigma>s_{2} \sigma$, we conclude that

$$
\begin{aligned}
\left\|f-\widehat{f}_{J}\right\|_{L^{2}\left(\Omega_{1} \times \Omega_{2}\right)}^{2} & \lesssim 2^{-2 J s_{2} \sigma}\{1+1\}\|f\|_{H_{\text {mix }}^{s_{1}, s_{2}}\left(\Omega_{1} \times \Omega_{2}\right)}^{2} \\
& \lesssim 2^{-2 J s_{2} \sigma}\|f\|_{H_{m i x}^{s_{1}, s_{2}}\left(\Omega_{1} \times \Omega_{2}\right)}^{2} .
\end{aligned}
$$

Finally, if $s_{1} / \sigma=s_{2} \sigma$, we have

$$
\begin{aligned}
\left\|f-\widehat{f}_{J}\right\|_{L^{2}\left(\Omega_{1} \times \Omega_{2}\right)}^{2} & \lesssim 2^{-2 J s_{2} \sigma}\left\{\sum_{j_{1}=0}^{J / \sigma} 1+1\right\}\|f\|_{H_{m i x}^{s_{1}, s_{2}}\left(\Omega_{1} \times \Omega_{2}\right)}^{2} \\
& \lesssim 2^{-2 J s_{2} \sigma} \frac{J}{\sigma}\|f\|_{H_{\text {mix }}^{s_{1}, s_{2}}\left(\Omega_{1} \times \Omega_{2}\right)}^{2}
\end{aligned}
$$

This completes the proof.

Remark 4.4. (i) The constant in estimate (4.12) depends again on the particular choice of $\sigma$.

(ii) As before, one readily verifies that the estimate (4.12) is essentially sharp. Nevertheless, if $s_{1}<r_{1}$ and $s_{2}<r_{2}$, then the factor $\sqrt{J}$ for the case $s_{1} / \sigma=s_{2} \sigma$ in (4.12) can be removed by using more sophisticated estimates; see [10, 21.

(iii) In the full tensor product space $V_{J / \sigma}^{(1)} \otimes V_{J \sigma}^{(2)}$, we obtain the error estimate $\inf _{f_{J} \in V_{J / \sigma}^{(1)} \otimes V_{J \sigma}^{(2)}}\left\|f-f_{J}\right\|_{L^{2}\left(\Omega_{1} \times \Omega_{2}\right)} \lesssim 2^{-J \min \left\{s_{1} / \sigma, s_{2} \sigma\right\}}\|f\|_{H_{m i x}^{s_{1}, 0}\left(\Omega_{1} \times \Omega_{2}\right) \cap H_{m i x}^{0, s_{2}}\left(\Omega_{1} \times \Omega_{2}\right)}$.

This gives, under the smoothness assumptions of Theorem 4.3. essentially the same rate of convergence as in (4.12). 
By combining Theorems 4.1 and 4.3 we can express the convergence rate in terms of the number of degrees of freedom $N:=\operatorname{dim} \widehat{V}_{J}^{\sigma}$. This gives us the cost complexity of approximating functions in the sparse tensor product spaces $\widehat{V}_{J}^{\sigma}$.

Corollary 4.5. Let $0<s_{1} \leq r_{1}, 0<s_{2} \leq r_{2}$ and $f \in H_{\text {mix }}^{s_{1}, s_{2}}\left(\Omega_{1} \times \Omega_{2}\right)$. Furthermore, denote by $N:=\operatorname{dim} \widehat{V}_{J}^{\sigma}$ the number of degrees of freedom in the sparse tensor product space $\widehat{V}_{J}^{\sigma}$ and set

$$
\alpha:=\frac{\min \left\{s_{1} / \sigma, s_{2} \sigma\right\}}{\max \left\{n_{1} / \sigma, n_{2} \sigma\right\}} .
$$

If $n_{1} / \sigma \neq n_{2} \sigma$, then the approximation (4.11) in $\widehat{V}_{J}^{\sigma}$ produces the following convergence rate in terms of the degrees of freedom $N$ :

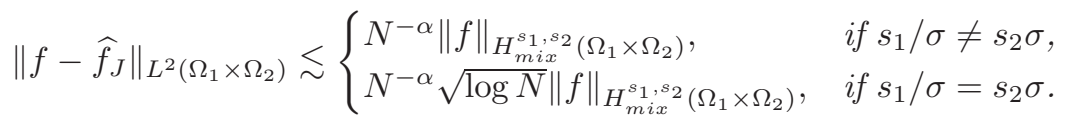

If $n_{1} / \sigma=n_{2} \sigma$, we have

$$
\left\|f-\widehat{f}_{J}\right\|_{L^{2}\left(\Omega_{1} \times \Omega_{2}\right)} \lesssim \begin{cases}N^{-\alpha}(\log N)^{\alpha}\|f\|_{H_{\text {mix }}^{s_{1}, s_{2}}\left(\Omega_{1} \times \Omega_{2}\right)}, & \text { if } s_{1} / \sigma \neq s_{2} \sigma, \\ N^{-\alpha}(\log N)^{\alpha+\frac{1}{2}}\|f\|_{H_{m i x}^{s_{1}, s_{2}}\left(\Omega_{1} \times \Omega_{2}\right)}, & \text { if } s_{1} / \sigma=s_{2} \sigma .\end{cases}
$$

Proof. If $n_{1} / \sigma \neq n_{2} \sigma$ we have $N \sim 2^{J \max \left\{n_{1} / \sigma, n_{2} \sigma\right\}}$ due to [4.7). Hence, it holds

$$
N^{-\alpha}=N^{-\frac{\min \left\{s_{1} / \sigma, s_{2} \sigma\right\}}{\max \left\{n_{1} / \sigma, n_{2} \sigma\right\}}} \sim 2^{-J \min \left\{s_{1} / \sigma, s_{2} \sigma\right\}}
$$

which, together with (4.12), yields the first error estimate.

If $n_{1} / \sigma=n_{2} \sigma$, then the sparse tensor product space $\widehat{V}_{J}^{\sigma}$ contains $N \sim 2^{J n_{2} \sigma} J=$ $2^{J \min \left\{n_{1} / \sigma, n_{2} \sigma\right\}} J$ degrees of freedom. Consequently, by noting that $J \sim \log (N / J) \lesssim$ $\log N$, we obtain from (4.12) the estimate

$$
\inf _{\widehat{f}_{J} \in \widehat{V}_{J}^{\sigma}}\left\|f-\widehat{f}_{J}\right\|_{L^{2}\left(\Omega_{1} \times \Omega_{2}\right)} \lesssim\left(\frac{N}{\log N}\right)^{-\alpha}\|f\|_{H_{m i x}^{s_{1}, s_{2}}\left(\Omega_{1} \times \Omega_{2}\right)}
$$

provided that $s_{1} / \sigma \neq s_{2} \sigma$.

In the case of $s_{1} / \sigma=s_{2} \sigma$, the additional factor $\sqrt{J} \lesssim \sqrt{\log N}$ needs to be inserted as a multiplicative factor. This completes the proof.

\section{Discussion of the Results}

5.1. Maximal regularity. In Corollary 4.5 we computed the cost complexities when approximating functions from $H_{m i x}^{s_{1}, s_{2}}\left(\Omega_{1} \times \Omega_{2}\right)$ in the sparse tensor product spaces $\widehat{V}_{J}^{\sigma}$ with arbitrary $\sigma>0$ and for $0<s_{1} \leq r_{1}$ and $0<s_{2} \leq r_{2}$. Now, we derive the cost complexity needed for the representation of a given function $f \in H_{m i x}^{r_{1}, r_{2}}\left(\Omega_{1} \times \Omega_{2}\right)$ with maximal regularity, i.e., for $s_{1}=r_{1}$ and $s_{2}=r_{2}$. Note that related results have been computed in [3, 9] for the particular situation of space-time discretizations of parabolic problems (i.e., $n_{1}=1, n_{2} \geq 1$ ).

In the following lemma we exclude the case $\frac{r_{1}}{n_{1}}=\frac{r_{2}}{n_{2}}$ to ensure $\frac{n_{1}}{\sigma} \neq n_{2} \sigma$ and $\frac{r_{1}}{\sigma} \neq r_{2} \sigma$.

Lemma 5.1. Assume that $\frac{r_{1}}{n_{1}} \neq \frac{r_{2}}{n_{2}}$ and let $\sigma$ be of the form

$$
\sigma=\sqrt{\frac{(1-\lambda) n_{1}+\lambda r_{1}}{(1-\lambda) n_{2}+\lambda r_{2}}}, \quad \lambda \in(0,1) .
$$


Then, the cost complexity to approximate a function $f \in H_{\text {mix }}^{r_{1}, r_{2}}\left(\Omega_{1} \times \Omega_{2}\right)$ in the sparse tensor product spaces $\widehat{V}_{J}^{\sigma}$ is

$$
\mathcal{O}\left(N^{\left.-\min \left\{\frac{r_{1}}{n_{1}}, \frac{r_{2}}{n_{2}}\right\}\right)}\right)
$$

Proof. Assume that $\frac{r_{1}}{n_{1}}<\frac{r_{2}}{n_{2}}$. Then, due to the inequalities

$$
\begin{gathered}
r_{1}\left((1-\lambda) n_{2}+\lambda r_{2}\right)<r_{2}\left((1-\lambda) n_{1}+\lambda r_{1}\right), \\
n_{1}\left((1-\lambda) n_{2}+\lambda r_{2}\right)>n_{2}\left((1-\lambda) n_{1}+\lambda r_{1}\right),
\end{gathered}
$$

it follows that

$$
\min \left\{\frac{r_{1}}{\sigma}, r_{2} \sigma\right\}=\frac{r_{1}}{\sigma} \text { and } \max \left\{\frac{n_{1}}{\sigma}, n_{2} \sigma\right\}=\frac{n_{1}}{\sigma} .
$$

Analogously, in the case $\frac{r_{1}}{n_{1}}>\frac{r_{2}}{n_{2}}$, it holds that

$$
\min \left\{\frac{r_{1}}{\sigma}, r_{2} \sigma\right\}=r_{2} \sigma \quad \text { and } \max \left\{\frac{n_{1}}{\sigma}, n_{2} \sigma\right\}=n_{2} \sigma .
$$

Consequently, from Corollary 4.5. for $s_{1}=r_{1}$ and $s_{2}=r_{2}$, the cost complexity is

$$
\mathcal{O}\left(N^{-\frac{\min \left\{r_{1} / \sigma, r_{2} \sigma\right\}}{\max \left\{n_{1} / \sigma, n_{2} \sigma\right\}}}\right)=\mathcal{O}\left(N^{-\min \left\{\frac{r_{1}}{n_{1}}, \frac{r_{2}}{n_{2}}\right\}}\right) .
$$

Remark 5.2. (i) For the left limit $\lambda=0$ it holds that $\sigma=\sqrt{n_{1} / n_{2}}$ and we have $n_{1} / \sigma=n_{2} \sigma=\sqrt{n_{1} n_{2}}$. Thus, the sparse tensor product space $\widehat{V}_{J}^{\sigma}$ contains $\mathcal{O}\left(2^{J \sqrt{n_{1} n_{2}}} J\right)$ degrees of freedom due to Theorem 4.1. Hence, the logarithmic term $(\log N)^{\min \left\{\frac{r_{1}}{n_{1}}, \frac{r_{2}}{n_{2}}\right\}}$ has to be inserted as a multiplicative factor into (5.14).

(ii) For the right limit $\lambda=1$ we have $\sigma=\sqrt{r_{1} / r_{2}}$ and thus $r_{1} / \sigma=r_{2} \sigma=\sqrt{r_{1} r_{2}}$. Then, with Theorem 4.3. we achieve only an accuracy of order $\mathcal{O}\left(2^{-J \sqrt{r_{1} r_{2}}} \sqrt{J}\right)$ in the sparse tensor product space $\widehat{V}_{J}^{\sigma}$. Thus, the logarithmic term $\sqrt{\log (N)}$ has to be inserted as a multiplicative factor into (5.14).

(iii) If $\frac{r_{1}}{n_{1}}=\frac{r_{2}}{n_{2}}$, we have $n_{1} / \sigma=n_{2} \sigma$ and $r_{1} / \sigma=r_{2} \sigma$ for all $\lambda=[0,1]$. Therefore, both types of logarithmic terms have to be inserted as a multiplicative factors into the cost complexity estimate (5.14) in this situation.

One readily verifies that $\sigma=\sigma(\lambda)$ from (5.13) satisfies the inequality

$$
\min \left\{\frac{r_{1}}{r_{2}}, \frac{n_{1}}{n_{2}}\right\} \leq \sigma^{2} \leq \max \left\{\frac{r_{1}}{r_{2}}, \frac{n_{1}}{n_{2}}\right\} \quad \text { for all } \lambda \in[0,1] .
$$

In particular, since the function $\sigma=\sigma(\lambda)$ is continuous in $\lambda$, each value between the upper and lower bound is admitted. Via Lemma 5.1 and Remark 5.2 we covered all such choices of $\sigma$.

In the following lemma we consider the remaining choices of $\sigma$, i.e., the case that the parameter $\sigma$ lies outside of the interval given by (5.15). We will prove that the convergence rate is then substantially smaller than that in (5.14). This result shows that all interesting parameters $\sigma$ are of the form (5.13). We therefore can restrict ourselves to such choices of $\sigma$ (cf. Figure 5.2). Note finally that the interval in 5.15 degenerates to the single point $\sigma^{2}=\frac{n_{1}}{n_{2}}$ in the case $\frac{r_{1}}{n_{1}}=\frac{r_{2}}{n_{2}}$.

Lemma 5.3. Let $\sigma$ be such that

$$
\sigma^{2}<\min \left\{\frac{r_{1}}{r_{2}}, \frac{n_{1}}{n_{2}}\right\} \quad \text { or } \quad \max \left\{\frac{r_{1}}{r_{2}}, \frac{n_{1}}{n_{2}}\right\}<\sigma^{2} .
$$




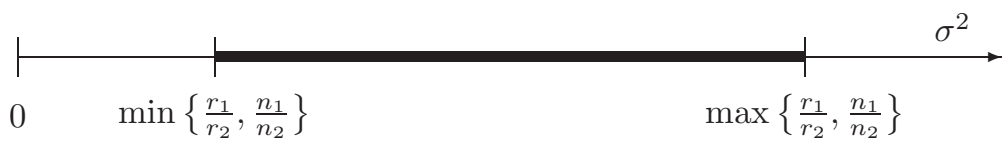

Figure 5.2. The optimal convergence rate is achieved for all $\sigma^{2}$ which are in the interval $\left(\min \left\{\frac{r_{1}}{r_{2}}, \frac{n_{1}}{n_{2}}\right\}, \max \left\{\frac{r_{1}}{r_{2}}, \frac{n_{1}}{n_{2}}\right\}\right)$. If $\sigma^{2}$ is an endpoint of this interval, logarithmic factors appear. The convergence rate is substantially smaller outside the interval.

Then, the convergence rate is substantially smaller than that in (5.14).

Proof. Under the assumption $\sigma^{2}<\min \left\{\frac{r_{1}}{r_{2}}, \frac{n_{1}}{n_{2}}\right\}$ it follows that

$$
\min \left\{\frac{r_{1}}{r_{2}}, \sigma^{2}\right\}=\sigma^{2} \quad \text { and } \max \left\{\frac{n_{1}}{n_{2}}, \sigma^{2}\right\}=\frac{n_{1}}{n_{2}} .
$$

Consequently, according to Corollary 4.5, the cost complexity is essentially, i.e., up to $\log N$-terms,

$$
\mathcal{O}\left(N^{-\frac{\min \left\{\frac{r_{1}}{\sigma}, r_{2} \sigma\right\}}{\max \left\{\frac{n_{1}}{\sigma}, n_{2} \sigma\right\}}}\right)=\mathcal{O}\left(N^{-\frac{\frac{r_{2}}{\sigma} \min \left\{\frac{r_{1}}{r_{2}}, \sigma^{2}\right\}}{\sigma} \max \left\{\frac{n_{1}}{n_{2}}, \sigma^{2}\right\}}\right)=\mathcal{O}\left(N^{-\frac{r_{2} \sigma^{2}}{n_{1}}}\right) .
$$

We therefore have to show that

$$
\frac{r_{2} \sigma^{2}}{n_{1}}<\min \left\{\frac{r_{1}}{n_{1}}, \frac{r_{2}}{n_{2}}\right\} .
$$

But this inequality follows immediately from

$$
\frac{r_{2} \sigma^{2}}{n_{1}}<\frac{r_{1}}{n_{1}} \Leftrightarrow \sigma^{2}<\frac{r_{1}}{r_{2}} \text { and } \frac{r_{2} \sigma^{2}}{n_{1}}<\frac{r_{2}}{n_{2}} \Leftrightarrow \sigma^{2}<\frac{n_{1}}{n_{2}} .
$$

In complete analogy the assertion is shown for the case $\max \left\{\frac{r_{1}}{r_{2}}, \frac{n_{1}}{n_{2}}\right\}<\sigma^{2}$ where the cost complexity is essentially $\mathcal{O}\left(N^{-r_{1} /\left(n_{2} \sigma^{2}\right)}\right)$.

5.2. Approximation of functions with anisotropic mixed Sobolev smoothness. If we consider the approximation of a function $f \in H_{\text {mix }}^{s_{1}, s_{2}}\left(\Omega_{1} \times \Omega_{2}\right)$ with arbitrary $0<s_{1} \leq r_{1}$ and $0<s_{2} \leq r_{2}$, then we get results which are completely analogous to the last subsection. The only difference is that $r_{1}$ has to be replaced by $s_{1}$ and $r_{2}$ has to be replaced by $s_{2}$. This means, for all $\sigma$ which are contained in the interval

$$
\min \left\{\frac{s_{1}}{s_{2}}, \frac{n_{1}}{n_{2}}\right\} \leq \sigma^{2} \leq \max \left\{\frac{s_{1}}{s_{2}}, \frac{n_{1}}{n_{2}}\right\}
$$

the error $\left\|f-\widehat{f_{J}}\right\|_{L^{2}\left(\Omega_{1} \times \Omega_{2}\right)}$ behaves essentially like

$$
\mathcal{O}\left(N^{-\min \left\{\frac{s_{1}}{n_{1}}, \frac{s_{2}}{n_{2}}\right\}}\right)
$$

For all $\sigma$ which are outside the above interval, the convergence rate is substantially smaller. Obviously, equilibrating the number of unknowns is now the best choice since $\sigma=\sqrt{n_{1} / n_{2}}$ is the only point which is always contained in the optimality interval given by (5.16). 
5.3. Approximation of functions with isotropic Sobolev smoothness. In the following discussion, we consider the approximation of an isotropic function $f \in H^{p}\left(\Omega_{1} \times \Omega_{2}\right)$. If $p \geq r_{1}+r_{2}$ we fall into the maximal regularity case, discussed in Subsection 5.1, since $H_{\text {mix }}^{r_{1}, r_{2}}\left(\Omega_{1} \times \Omega_{2}\right) \subset H^{r_{1}+r_{2}}\left(\Omega_{1} \times \Omega_{2}\right)$.

We therefore may assume that $p<r_{1}+r_{2}$. The highest rate of convergence is then achieved if the minimum of $s_{1} / \sigma$ and $s_{2} \sigma$ constrained to $s_{1}+s_{2}=p$ is maximal. Since the maximum is attained if $s_{1} / \sigma=s_{2} \sigma$, we arrive at the choice

$$
s_{1}:=\min \left\{\frac{p \sigma^{2}}{\sigma^{2}+1}, r_{1}\right\}, \quad s_{2}:=\min \left\{\frac{p}{\sigma^{2}+1}, r_{2}\right\} .
$$

We then have the following rates which depend on $\sigma$ :

Theorem 5.4. Let $f \in H^{p}\left(\Omega_{1} \times \Omega_{2}\right)$ be a given function and let $\sigma$ be a fixed, given number which satisfies (5.13). Then, as long as

$$
\frac{p \sigma}{\sigma^{2}+1} \geq \min \left\{\frac{r_{1}}{\sigma}, r_{2} \sigma\right\}
$$

the function $f$ can be approximated in $\widehat{V}_{J}^{\sigma}$ with the maximal convergence rate as specified in Lemma 5.1 and Remark 5.2. Otherwise, we get essentially, i.e., up to $\log N$-terms, the reduced cost complexity $\mathcal{O}\left(N^{-\beta}\right)$ with

$$
\beta=\frac{p}{n_{1}} \frac{(1-\lambda) n_{1}+\lambda r_{1}}{(1-\lambda)\left(n_{1}+n_{2}\right)+\lambda\left(r_{1}+r_{2}\right)} \quad \text { if } \frac{r_{1}}{n_{1}} \leq \frac{r_{2}}{n_{2}}
$$

and

$$
\beta=\frac{p}{n_{2}} \frac{(1-\lambda) n_{2}+\lambda r_{2}}{(1-\lambda)\left(n_{1}+n_{2}\right)+\lambda\left(r_{1}+r_{2}\right)} \quad \text { if } \frac{r_{1}}{n_{1}}>\frac{r_{2}}{n_{2}} .
$$

Proof. Choose $s_{1}, s_{2}$ as in (5.17). Then, we have

$$
\min \left\{\frac{s_{1}}{\sigma}, s_{2} \sigma\right\}=\min \left\{\frac{p \sigma}{\sigma^{2}+1}, \frac{r_{1}}{\sigma}, r_{2} \sigma\right\} .
$$

Thus, if (5.18) holds, the assertion is obvious due to

$$
\min \left\{\frac{s_{1}}{\sigma}, s_{2} \sigma\right\}=\min \left\{\frac{r_{1}}{\sigma}, r_{2} \sigma\right\}
$$

and Corollary 4.5. We therefore may assume that

$$
\frac{p \sigma}{\sigma^{2}+1}<\min \left\{\frac{r_{1}}{\sigma}, r_{2} \sigma\right\}
$$

which implies

$$
\min \left\{\frac{s_{1}}{\sigma}, s_{2} \sigma\right\}=\frac{p \sigma}{\sigma^{2}+1} .
$$

In the case of $\frac{r_{1}}{n_{1}} \leq \frac{r_{2}}{n_{2}}$ it follows from (5.13) that $\max \left\{n_{1} / \sigma, n_{2} \sigma\right\}=n_{1} / \sigma$ and thus the cost complexity is essentially

$$
\mathcal{O}\left(N^{-\frac{\frac{p \sigma}{1+\sigma^{2}}}{\max \left\{\frac{n_{1}}{\sigma}, n_{2} \sigma\right\}}}\right)=\mathcal{O}\left(N^{-\frac{p}{n_{1}} \frac{(1-\lambda) n_{1}+\lambda r_{1}}{(1-\lambda)\left(n_{1}+n_{2}\right)+\lambda\left(r_{1}+r_{2}\right)}}\right) .
$$

Vice versa, if $\frac{r_{1}}{n_{1}}>\frac{r_{2}}{n_{2}}$, it holds that $\max \left\{n_{1} / \sigma, n_{2} \sigma\right\}=n_{2} \sigma$ and consequently the cost complexity is essentially

$$
\mathcal{O}\left(N^{-\frac{p \sigma}{\max \left\{\frac{n_{1}}{\sigma}, n_{2} \sigma\right\}}}\right)=\mathcal{O}\left(N^{-\frac{p}{n_{2}} \frac{(1-\lambda) n_{2}+\lambda r_{2}}{(1-\lambda)\left(n_{1}+n_{2}\right)+\lambda\left(r_{1}+r_{2}\right)}}\right) .
$$


This completes the proof.

Remark 5.5. For $\sigma=\sqrt{r_{1} / r_{2}}$, that is $\lambda=1$, the choice of $s_{1}, s_{2}$ from (5.17) leads to $s_{1}=r_{1} \min \left\{\frac{p}{r_{1}+r_{2}}, 1\right\}=r_{1} \frac{p}{r_{1}+r_{2}}$ and $s_{2}=r_{2} \min \left\{\frac{p}{r_{1}+r_{2}}, 1\right\}=r_{2} \frac{p}{r_{1}+r_{2}}$ provided that $p<r_{1}+r_{2}$. Therefore, (5.18) is never satisfied if $p<r_{1}+r_{2}$ and we always get the reduced cost complexity $\mathcal{O}\left(N^{-\gamma}\right)$ with

$$
\gamma=\frac{\sqrt{r_{1} r_{2}} \frac{p}{r_{1}+r_{2}}}{\max \left\{\frac{n_{1}}{\sigma}, n_{2} \sigma\right\}}=-\frac{p}{r_{1}+r_{2}} \min \left\{\frac{r_{1}}{n_{1}}, \frac{r_{2}}{n_{2}}\right\}
$$

which is equivalent to (5.19) and (5.20), respectively.

The following result shows that, as long as the convergence rate is not maximal, the best setting to approximate an isotropic function is provided by the choice $\sigma=\sqrt{n_{1} / n_{2}}$.

Proposition 5.6. Let $f \in H^{p}\left(\Omega_{1} \times \Omega_{2}\right)$ be a given function with arbitrary $0<$ $p<r_{1}+r_{2}$ and $\sigma$ as in (5.13). Then, the approximation power of $\widehat{V}_{J}^{\sigma}$ is better, the smaller the parameter $\lambda$ is. The highest convergence rate results, therefore, for the choice $\lambda=0$, i.e., $\sigma=\sqrt{n_{1} / n_{2}}$, while the lowest convergence rate results from the choice $\lambda=1$, i.e., $\sigma=\sqrt{r_{1} / r_{2}}$.

Proof. To prove the assertion it suffices to show that, for fixed $p<r_{1}+r_{2}$, the convergence rates (5.19) and (5.20) decrease as the parameter $\lambda$ increases.

Assume first that $\frac{r_{1}}{n_{1}}<\frac{r_{2}}{n_{2}}$. Then, the function

$$
g(\lambda):=\frac{(1-\lambda) n_{1}+\lambda r_{1}}{(1-\lambda)\left(n_{1}+n_{2}\right)+\lambda\left(r_{1}+r_{2}\right)}
$$

is monotonically decreasing due to

$$
g(\lambda)^{\prime}=\frac{r_{1} n_{2}-n_{1} r_{2}}{\left((1-\lambda)\left(n_{1}+n_{2}\right)+\lambda\left(r_{1}+r_{2}\right)\right)^{2}}<0 .
$$

Hence, for $\lambda<\mu$, we have

$$
\frac{p}{n_{1}} \frac{(1-\lambda) n_{1}+\lambda r_{1}}{(1-\lambda)\left(n_{1}+n_{2}\right)+\lambda\left(r_{1}+r_{2}\right)}>\frac{p}{n_{1}} \frac{(1-\mu) n_{1}+\mu r_{1}}{(1-\mu)\left(n_{1}+n_{2}\right)+\mu\left(r_{1}+r_{2}\right)}
$$

which, in view of (5.19), is the first part of the assertion.

Likewise, it holds that

$$
h(\lambda):=\frac{(1-\lambda) n_{2}+\lambda r_{2}}{(1-\lambda)\left(n_{1}+n_{2}\right)+\lambda\left(r_{1}+r_{2}\right)}
$$

is monotonically decreasing provided that $\frac{r_{1}}{n_{1}}>\frac{r_{2}}{n_{2}}$. This gives

$$
\frac{p}{n_{2}} \frac{(1-\lambda) n_{2}+\lambda r_{2}}{(1-\lambda)\left(n_{1}+n_{2}\right)+\lambda\left(r_{1}+r_{2}\right)}>\frac{p}{n_{2}} \frac{(1-\mu) n_{2}+\mu r_{2}}{(1-\mu)\left(n_{1}+n_{2}\right)+\mu\left(r_{1}+r_{2}\right)}
$$

for $\lambda<\mu$. We then obtain the second part of the assertion with (5.20).

Remark 5.7. It is well known that the highest rate, which is achieved by full tensor product spaces, is given by Kolmogorov's $n$-width; see [15]. If $r_{1}=r_{2}$, it is $N^{-p /\left(n_{1}+n_{2}\right)}$, i.e., $N \lesssim \varepsilon^{-\left(n_{1}+n_{2}\right) / p}$, for Sobolev balls in $H^{p}$ provided that $p \leq r_{1}$. From Theorem 5.4 it follows that the sparse grid space $\widehat{V}_{J}^{\sigma}$ with $\sigma=\sqrt{n_{1} / n_{2}}$ also achieves this rate up to logarithmic terms. Note finally that in the case $p>r_{1}$ our construction implicitly exploits the then appearing mixed regularity and thus obtains better rates. 


\section{NumericAl EXPERIMENTS}

We now present the results of our numerical experiments. To this end, we consider smooth and nonsmooth model functions. The practically obtained rates are in good agreement with the presented theory.

6.1. An alternative decomposition of the sparse grid space. First, we give a hint on an alternative decomposition of $\widehat{V}_{J}^{\sigma}$, which allows a simpler implementation in our case of two-fold tensor-product domains. On $\Omega_{1}$ we use wavelets for the discretization which gives rise to a decomposition

$$
V_{J / \sigma}^{(1)}=\bigoplus_{j=0}^{J / \sigma} W_{j}^{(1)} \subset L^{2}((0,1)),
$$

i.e., we have the sequence

$$
W_{0}^{(1)}, \quad W_{1}^{(1)}, \quad \cdots \quad, W_{J / \sigma}^{(1)} .
$$

On $\Omega_{2}$ we apply finite elements with sufficient polynomial exactness on a series of hierarchical triangular meshes which stem from uniform refinement. They generate a family of nested single-scale spaces

$$
V_{0}^{(2)} \subset V_{1}^{(2)} \subset \cdots \subset V_{J \sigma}^{(2)} \subset L^{2}\left((0,1)^{2}\right) .
$$

We define the sparse grid space $\widehat{V}_{J}^{\sigma}$ by

$$
\widehat{V}_{J}^{\sigma}:=\bigoplus_{j_{1} \sigma+j_{2} / \sigma=J} W_{j_{1}}^{(1)} \otimes V_{j_{2}}^{(2)} .
$$

This definition looks different from that in (3.6). But it is indeed equivalent since

$$
\begin{gathered}
\widehat{V}_{J}^{\sigma}=\bigoplus_{j_{1} \sigma+j_{2} / \sigma \leq J} W_{j_{1}}^{(1)} \otimes W_{j_{2}}^{(2)}=\bigoplus_{j_{1}=0}^{J / \sigma} W_{j_{1}}^{(1)} \otimes(\underbrace{\bigoplus_{j_{2}=0}^{J \sigma-j_{1} \sigma^{2}} W_{j_{2}}^{(2)}}_{=V_{J \sigma-j_{1} \sigma^{2}}^{(2)}}) \\
=\bigoplus_{j_{1} \sigma+j_{2} / \sigma=J} W_{j_{1}}^{(1)} \otimes V_{j_{2}}^{(2)} .
\end{gathered}
$$

This way, standard finite element discretizations for the sequence (6.22) can directly be employed and the need of a wavelet construction on $\Omega_{2}$ is avoided. This significantly simplifies programming since it allows, at least for problems involving product operators, the reuse of an existing FEM-code on $\Omega_{2}$, makes the use of standard multilevel solvers directly possible and helps to circumvent the difficulties a direct wavelet discretization may pose, e.g., for the treatment of boundary conditions there.

6.2. Smooth functions. We consider the situation of $\Omega_{1}=(0,1)$ and $\Omega_{2}=(0,1)^{2}$, that is, $n_{1}=1$ and $n_{2}=2$. On $\Omega_{1}$ we use piecewise constant (i.e., $r_{1}=1$ ) or linear (i.e., $r_{1}=2$ ) wavelets for the discretization which gives rise to a decomposition (6.21). On $\Omega_{2}$ we apply either piecewise constant (i.e., $r_{2}=1$ ) or globally continuous, piecewise linear (i.e., $r_{2}=2$ ) finite elements on a series of hierarchical triangular meshes which stem from uniform refinement. They generate the family 

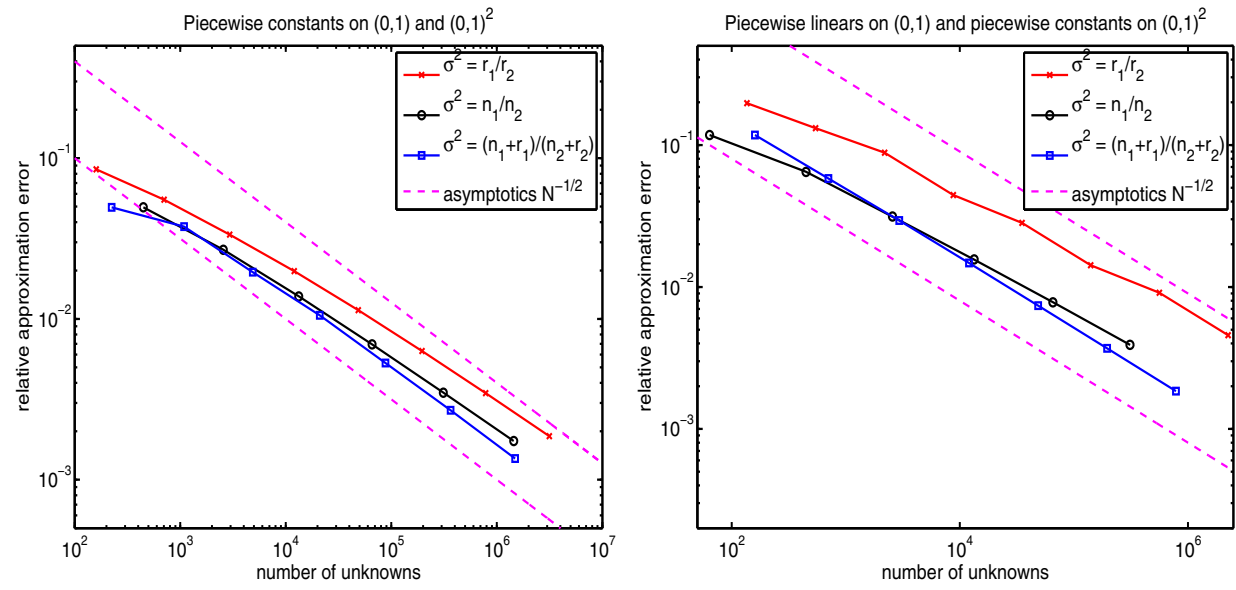

FiguRE 6.3. Cost complexities for approximating a smooth function in case of $n_{1}=1, n_{2}=2$ and $r_{1}=r_{2}=1$ (left) or $r_{1}=2$, $r_{2}=1$ (right).

of nested single-scale spaces $\left\{V_{j}^{(2)}\right\}$. We then employ the decomposition (6.23) for the approximation in $L^{2}\left(\Omega_{1} \times \Omega_{2}\right)$.

For different choices of $r_{1}, r_{2}$, and $\sigma$, we compute the approximation error of the sparse grid interpolant of the $C^{\infty}$-function

$$
f(x, \mathbf{y})=\sin \left(y_{1}\right)+\sin ^{2}(2 \pi x)\left(y_{1}+y_{2}\right) y_{2} .
$$

We first apply piecewise constant finite elements for the sequence $\left\{V_{j}^{(2)}\right\}$ on $\Omega_{2}$ in $\widehat{V}_{J}^{\sigma}$. In Figure 6.3, we plot the measured approximation error versus the number of degrees of freedom for the three values $\sigma=\sqrt{r_{1} / r_{2}}, \sigma=\sqrt{n_{1} / n_{2}}$, and $\sigma=\sqrt{\left(r_{1}+n_{1}\right) /\left(r_{2}+n_{2}\right)}$. On the interval $\Omega_{1}$ we employ piecewise constant (left plot) and piecewise linear (right plot) wavelets. All the curves are quite similar and behave essentially like $N^{-1 / 2}$ (indicated by the dashed line) as predicted by Lemma 5.1

The best cost complexity rate is observed for the cost-benefit equilibrated sparse grid space, i.e., for $\sigma=\sqrt{\left(r_{1}+n_{1}\right) /\left(r_{2}+n_{2}\right)}$, which is in accordance with Remark 5.2. It indeed seems to be linear with $N^{-1 / 2}$ asymptotics whereas the cost complexity rates for $\sigma=\sqrt{r_{1} / r_{2}}$ and $\sigma=\sqrt{n_{1} / n_{2}}$ exhibit additional logarithmic factors as stated in Remark 5.2 (ii) and (iii). This holds for both cases, i.e., $r_{1}=r_{2}=1$ and $r_{1}=2, r_{2}=1$.

Analogous observations are made if we employ piecewise linear wavelets on $\Omega_{1}$ and globally continuous, piecewise linear finite elements on $\Omega_{2}$. The results are given in the right plot of Figure 6.4. Here, the predicted asymptotics is $N^{-1}$, indicated by the dashed line.

If we combine globally continuous, piecewise linear finite elements $\left\{V_{j}^{(2)}\right\}$ on $\Omega_{2}$ with piecewise constant wavelets on $\Omega_{1}$, we are in the situation that $\sqrt{n_{1} / n_{2}}=$ $\sqrt{r_{1} / r_{2}}=\sqrt{\left(n_{1}+r_{1}\right) /\left(n_{2}+r_{2}\right)}$. Thus, the choice $\sigma=\sqrt{n_{1} / n_{2}}$ will lead to the best complexity, i.e., an essential convergence rate of $N^{-1}$. This is also seen from the left plot of Figure 6.4 where the approximation error is plotted versus the 

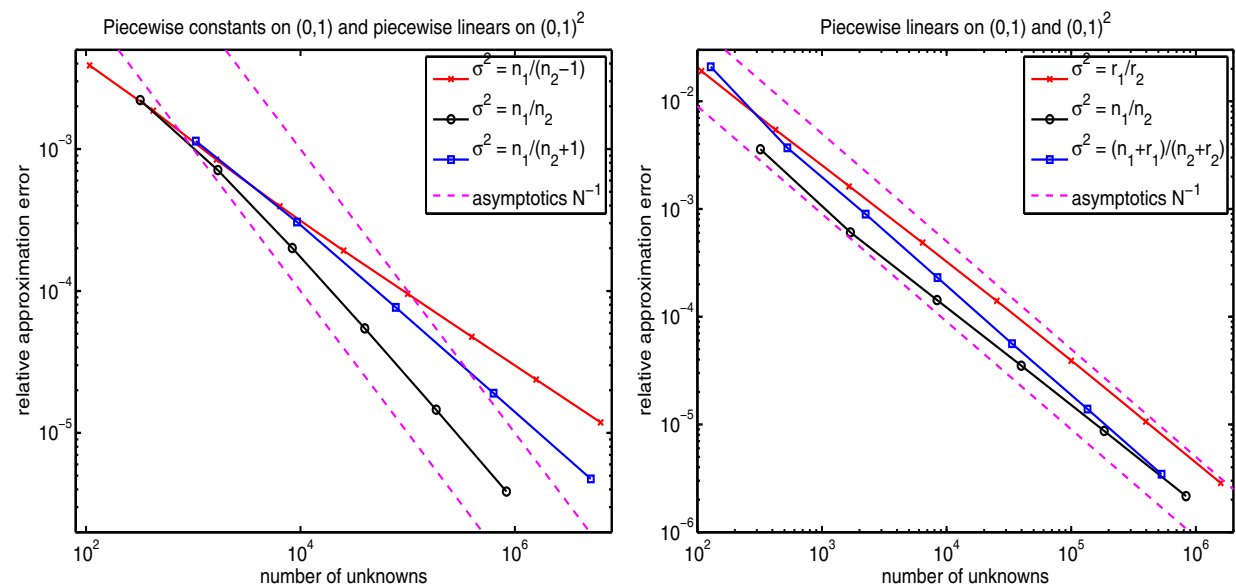

Figure 6.4. Cost complexities for approximating a smooth function in case of $n_{1}=1, n_{2}=2$ and $r_{1}=1, r_{2}=2$ (left) or $r_{1}=r_{2}=2$ (right).

TABLE 6.1. The predicted rates of convergence.

\begin{tabular}{|c|ccc|}
\hline ansatz functions & $\sigma=\sqrt{\frac{n_{1}}{n_{2}}}$ & $\sigma=\sqrt{\frac{r_{1}+n_{1}}{r_{2}+n_{2}}}$ & $\sigma=\sqrt{\frac{r_{1}}{r_{2}}}$ \\
\hline$r_{1}=r_{2}=1$ & $N^{-1 / 2}$ & $N^{-9 / 20}$ & $N^{-3 / 8}$ \\
$r_{1}=1, r_{2}=2$ & $N^{-1 / 2}$ & $N^{-1 / 2}$ & $N^{-1 / 2}$ \\
$r_{1}=2, r_{2}=1$ & $N^{-1 / 2}$ & $N^{-3 / 8}$ & $N^{-1 / 4}$ \\
$r_{1}=r_{2}=2$ & $N^{-1 / 2}$ & $N^{-3 / 7}$ & $N^{-3 / 8}$ \\
\hline
\end{tabular}

number of degrees of freedom in case of $\sigma=\sqrt{n_{1} /\left(n_{2}-1\right)}, \sigma=\sqrt{n_{1} / n_{2}}$, and $\sigma=\sqrt{n_{1} /\left(n_{2}+1\right)}$. Indeed, for $\sigma \neq \sqrt{n_{1} / n_{2}}$ a substantially smaller convergence rate is observed.

6.3. Nonsmooth functions. We now investigate the approximation rates for the function

$$
f(x, \mathbf{y})=\left|\sin (\pi x)-y_{1}\right| .
$$

One readily verifies that $f$ is in $H^{s}\left(\Omega_{1} \times \Omega_{2}\right)$ for all $s<3 / 2$. We apply the same ansatz functions on $\Omega_{1}$ and $\Omega_{2}$ as in the previous subsection and measure the approximation error of the sparse grid interpolant for the three values $\sigma=\sqrt{r_{1} / r_{2}}$, $\sigma=\sqrt{n_{1} / n_{2}}$, and $\sigma=\sqrt{\left(r_{1}+n_{1}\right) /\left(r_{2}+n_{2}\right)}$. If we insert the limit case $p:=3 / 2$, Theorem 5.4 predicts the convergence rates given in Table 6.1

The errors which were measured in our numerical calculations are plotted in Figures 6.5 and 6.6. The predicted convergence rates from Table 6.1 are visualized there as dashed lines. Figure 6.5 shows the case $r_{1}=r_{2}=1$ (left plot) and $r_{1}=2$, $r_{2}=1$ (right plot), Figure 6.6 shows the case $r_{1}=1, r_{2}=2$ (left plot) and $r_{1}=r_{2}=2$ (right plot). Equilibrating the number of degrees of freedom (that is, the choice $\left.\sigma:=\sqrt{n_{1} / n_{2}}\right)$ gives in any case the best rate $N^{-1 / 2}$. Note that this is even the same rate as for smooth functions if there we would apply piecewise constant functions on $\Omega_{1}$ and $\Omega_{2}$. As it can be seen in Figures 6.5 and 6.6 this rate 

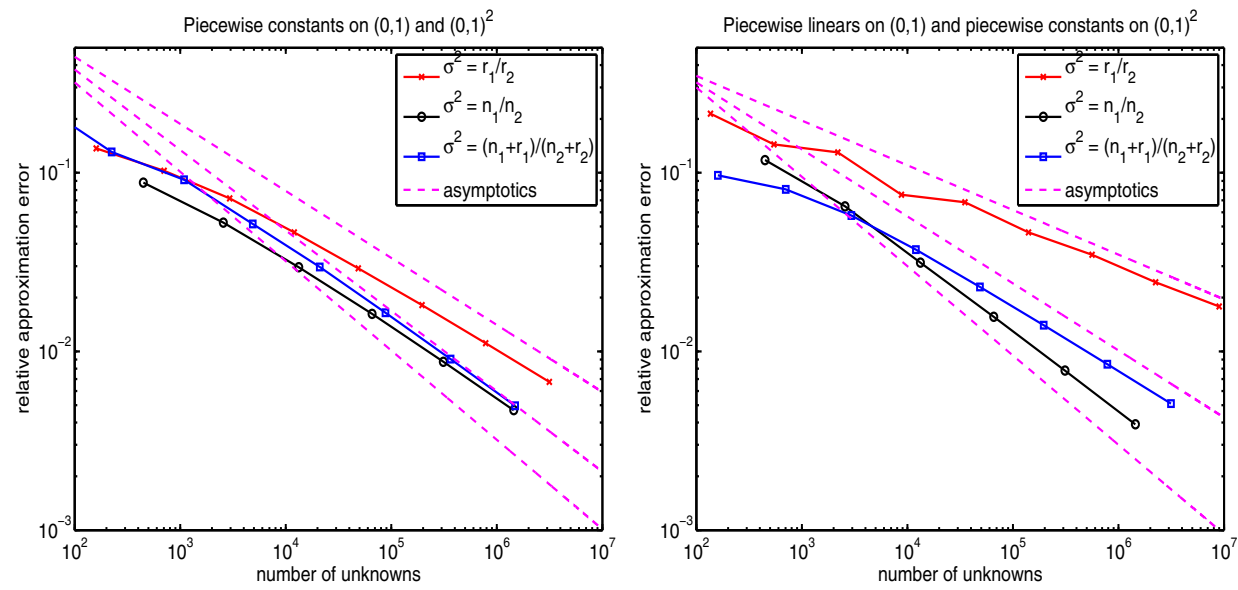

FiguRE 6.5. Cost complexities for approximating a nonsmooth function in case of $n_{1}=1, n_{2}=2$ and $r_{1}=r_{2}=1$ (left) or $r_{1}=2, r_{2}=1$ (right). The three asymptotic approximation rates are found in Table 6.1, respectively.
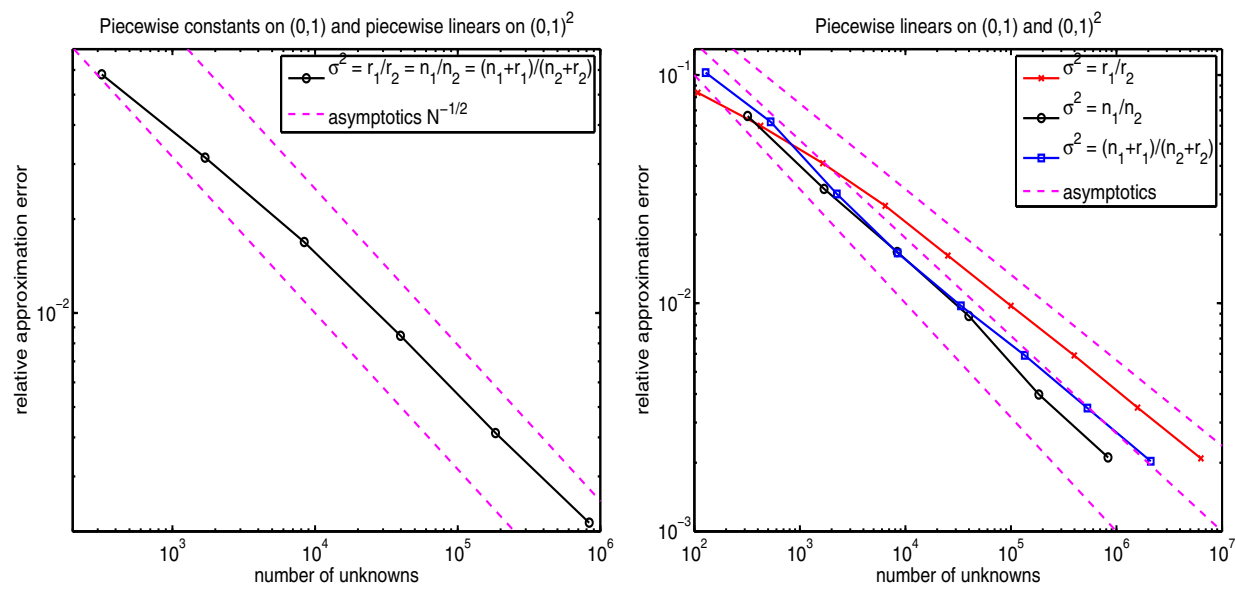

Figure 6.6. Cost complexities for approximating a nonsmooth function in case of $n_{1}=1, n_{2}=2$ and $r_{1}=1, r_{2}=2$ (left) or $r_{1}=r_{2}=2$ (right).

is essentially achieved. It is also seen that the choice $\sigma:=\sqrt{r_{1} / r_{2}}$ always gives a substantially smaller convergence rate. For $\sigma:=\sqrt{\left(r_{1}+n_{1}\right) /\left(r_{2}+n_{2}\right)}$ the rate is somewhere in between. However, its slope cannot be clearly seen from the plots except in the case $r_{1}=2$ and $r_{2}=1$. There, also the related asymptotics $N^{-3 / 8}$ is substantially different from $N^{-1 / 2}$. 


\section{Approximation in arbitrary nORMS}

So far, we only considered the case of the approximation error measured in the $L^{2}\left(\Omega_{1} \times \Omega_{2}\right)$-norm, i.e., the $H_{m i x}^{q_{1}, q_{2}}\left(\Omega_{1} \times \Omega_{2}\right)$-norm with $q_{1}=q_{2}=0$. However, certain applications involve other error norms which are of mixed type with more general $q_{1}, q_{2}$. For example, the two-point correlation of solutions to elliptic partial differential equations of second order with stochastic right-hand side are in $H_{m i x}^{1,1}\left(\Omega_{1} \times \Omega_{2}\right)$ (cf. [20]). Moreover, radiative transfer (cf. [23]) and homogenization problems (cf. [14]) lead naturally to the $H_{m i x}^{1,0}\left(\Omega_{1} \times \Omega_{2}\right)$-norm. Therefore, we comment on the situation where the error is considered in the $H_{\text {mix }}^{q_{1}, q_{2}}\left(\Omega_{1} \times \Omega_{2}\right)$-norm, with $-\widetilde{\gamma}_{1}<q_{1}<\gamma_{1}$, and $-\widetilde{\gamma}_{2}<q_{2}<\gamma_{2}$. Here, $\gamma_{i}$ and $\widetilde{\gamma}_{i}$ denotes the regularity of the primal and dual wavelets, respectively 2

First, in analogy to (2.3), we assume the shifted approximation properties

$$
\inf _{v_{j} \in V_{j}^{(i)}}\left\|u-v_{j}\right\|_{H^{q_{i}\left(\Omega_{i}\right)}} \lesssim 2^{-j\left(s_{i}-q_{i}\right)}\|u\|_{H^{s_{i}\left(\Omega_{i}\right)}}, \quad u \in H^{s_{i}}\left(\Omega_{i}\right)
$$

to hold for $q_{i} \leq s_{i} \leq r_{i}$ uniformly in $j$. In particular, for any $f \in H^{s_{i}}\left(\Omega_{i}\right)$, this induces the shifted estimate

$$
\left\|Q_{j}^{(i)} f\right\|_{H^{q_{i}\left(\Omega_{i}\right)}} \lesssim 2^{-j\left(s_{i}-q_{i}\right)}\|f\|_{H^{s_{i}\left(\Omega_{i}\right)}}, \quad q_{i} \leq s_{i} \leq r_{i} .
$$

This gives us straightforwardly the following generalization of Theorem 4.3 .

Theorem 7.1. Let $q_{1}<s_{1} \leq r_{1}, q_{2}<s_{2} \leq r_{2}$ and $f \in H_{\text {mix }}^{s_{1}, s_{2}}\left(\Omega_{1} \times \Omega_{2}\right)$. Then, the approximation

$$
\widehat{f}_{J}=\sum_{j_{1} \sigma+\frac{j_{2}}{\sigma} \leq J}\left(Q_{j_{1}}^{(1)} \otimes Q_{j_{2}}^{(2)}\right) f \in \widehat{V}_{J}^{\sigma}
$$

satisfies

$$
\begin{aligned}
& \left\|f-\widehat{f}_{J}\right\|_{H_{m i x}^{q_{1}, q_{2}}\left(\Omega_{1} \times \Omega_{2}\right)}
\end{aligned}
$$

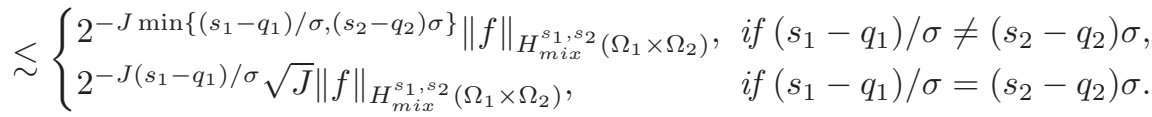

Proof. It follows by standard product arguments from (7.24) that

$$
\begin{aligned}
\left\|f-\widehat{f}_{J}\right\|_{H_{m i x}^{q_{1}, q_{2}}\left(\Omega_{1} \times \Omega_{2}\right)}^{2} & \lesssim \sum_{j_{1} \sigma+\frac{j_{2}}{\sigma}>J}\left\|\left(Q_{j_{1}}^{(1)} \otimes Q_{j_{2}}^{(2)}\right) f\right\|_{H_{m i x}^{q_{1}, q_{2}}(\Omega)}^{2} \\
& \lesssim \sum_{j_{1} \sigma+\frac{j_{2}}{\sigma}>J} 2^{-2\left(\left(s_{1}-q_{1}\right) j_{1}+\left(s_{2}-q_{2}\right) j_{2}\right)}\|f\|_{H_{m i x}^{s_{1}, s_{2}}\left(\Omega_{1} \times \Omega_{2}\right)}^{2} .
\end{aligned}
$$

The desired result follows from this in complete analogy to the lines of the proof of Theorem 4.3 with now just $s_{1}, s_{2}$ shifted to $s_{1}-q_{1}, s_{2}-q_{2}$ in the decay rates and clauses (but of course not in the smoothness assumption $\left.\|f\|_{H_{m i x}^{s_{1}, s_{2}}\left(\Omega_{1} \times \Omega_{2}\right)}\right)$.

\footnotetext{
${ }^{2}$ Here, we assume as usual that the polynomial exactness $r_{i}$ and $\widetilde{r_{i}}$ of the primal and dual wavelets is higher than their regularity $\gamma_{i}$ and $\widetilde{\gamma}_{i}$. If this is not the case, then the lower and upper bounds of the $q_{i}$ have to be correspondingly replaced by $-\widetilde{r_{i}}$ and $r_{i}$, compare also [5].
} 
Also, Corollary 4.5 directly carries over. We obtain:

Corollary 7.2. Let $q_{1}<s_{1} \leq r_{1}, q_{2}<s_{2} \leq r_{2}$ and $f \in H_{\operatorname{mix}}^{s_{1}, s_{2}}\left(\Omega_{1} \times \Omega_{2}\right)$. Furthermore, denote by $N:=\operatorname{dim} \widehat{V}_{J}^{\sigma}$ the number of degrees of freedom in the sparse tensor product space $\widehat{V}_{J}^{\sigma}$ and set

$$
\alpha:=\frac{\min \left\{\left(s_{1}-q_{1}\right) / \sigma,\left(s_{2}-q_{2}\right) \sigma\right\}}{\max \left\{n_{1} / \sigma, n_{2} \sigma\right\}} .
$$

If $n_{1} / \sigma \neq n_{2} \sigma$, then the approximation (4.11) in $\widehat{V}_{J}^{\sigma}$ produces the following convergence rate in terms of the degrees of freedom $N$ :

$$
\begin{aligned}
& \left\|f-\widehat{f}_{J}\right\|_{H_{m i x}^{q_{1}, q_{2}}\left(\Omega_{1} \times \Omega_{2}\right)} \\
& \lesssim \begin{cases}N^{-\alpha}\|f\|_{H_{\text {mix }}^{s_{1}, s_{2}}\left(\Omega_{1} \times \Omega_{2}\right)}, & \text { if }\left(s_{1}-q_{1}\right) / \sigma \neq\left(s_{2}-q_{2}\right) \sigma, \\
N^{-\alpha} \sqrt{\log N\|f\|_{H_{m i x}^{s_{1}, s_{2}}\left(\Omega_{1} \times \Omega_{2}\right)},}, & \text { if }\left(s_{1}-q_{1}\right) / \sigma=\left(s_{2}-q_{2}\right) \sigma .\end{cases}
\end{aligned}
$$

If $n_{1} / \sigma=n_{2} \sigma$, we have

$$
\begin{aligned}
\| f- & \widehat{f}_{J} \|_{H_{m i x}^{q_{1}, q_{2}}\left(\Omega_{1} \times \Omega_{2}\right)} \\
& \lesssim \begin{cases}N^{-\alpha}(\log N)^{\alpha}\|f\|_{H_{\text {mix }}^{s_{1}, s_{2}}\left(\Omega_{1} \times \Omega_{2}\right)}, & \text { if }\left(s_{1}-q_{1}\right) / \sigma \neq\left(s_{2}-q_{2}\right) \sigma, \\
N^{-\alpha}(\log N)^{\alpha+\frac{1}{2}}\|f\|_{H_{\text {mix }}^{s_{1}, s_{2}}\left(\Omega_{1} \times \Omega_{2}\right)}, & \text { if }\left(s_{1}-q_{1}\right) / \sigma=\left(s_{2}-q_{2}\right) \sigma .\end{cases}
\end{aligned}
$$

Furthermore, the discussion of the results in Subsections 5.1 and 5.2 also carries directly over with the obvious substitutions $\left\|f-\widehat{f}_{J}\right\|_{H_{m i x}^{q_{1}, q_{2}}\left(\Omega_{1} \times \Omega_{2}\right)}$ for $\| f-$ $\widehat{f}_{J} \|_{L^{2}\left(\Omega_{1} \times \Omega_{2}\right)}$ and $r_{1}-q_{1}, r_{2}-q_{2}$ for $r_{1}, r_{2}$ or $s_{1}-q_{1}, s_{2}-q_{2}$ for $s_{1}, s_{2}$ (in the rates and case clauses but of course not in the smoothness prerequisites $\|f\|_{H_{m i x}^{r_{1}, r_{2}}\left(\Omega_{1} \times \Omega_{2}\right)}$ and $\|f\|_{H_{m i x}^{s_{1}, s_{2}}\left(\Omega_{1} \times \Omega_{2}\right)}$, respectively).

\section{Concluding REmarks}

In the present paper we restricted ourselves to the construction of sparse tensor product spaces on two-fold tensor product domains. We now comment on the situation in the case of $m$-fold tensor product domains $\bigotimes_{i=1}^{m} \Omega_{i}$ with $\Omega_{i} \in \mathbb{R}^{n_{i}}, n_{i} \in$ $\mathbb{N}$, and associated multiscale hierarchies $\left\{V_{j}^{(i)}\right\}$ of polynomial exactness $r_{i}$.

It becomes clear from the theory presented in Section 4 that, when building the sparse tensor product space $\widehat{V}_{J}$ which consists of all tensor products $W_{j_{1}}^{(1)} \otimes \cdots \otimes$ $W_{j_{m}}^{(m)}$ whose indices satisfy the inequality $\sum_{i=1}^{m} j_{i} \cdot \alpha_{i} \leq J\left(\alpha_{i}>0\right)$, the approximation power is essentially the minimum of the approximation powers of the extremal univariate single-scale spaces $V_{J / \alpha_{i}}^{(i)}$. Thus, the approximation power in $\widehat{V}_{J}$ is essentially of the order $\mathcal{O}\left(2^{-J \min _{i \in\{1, \ldots, m\}}\left\{r_{i} / \alpha_{i}\right\}}\right)$ provided that the function to be approximated is smooth enough. If only $H^{s_{i}}\left(\Omega_{i}\right)$-smoothness with $s_{i} \leq r_{i}$ is provided in the $i$-th variable, the related rate has to be replaced by $\mathcal{O}\left(2^{-J \min _{i \in\{1, \ldots, m\}}\left\{s_{i} / \alpha_{i}\right\}}\right)$.

Furthermore, it is obvious that the number of unknowns in $\widehat{V}_{J}$ scales essentially like the maximum of the unknowns of the extremal univariate single-scale spaces, i.e., $\widehat{V}_{J}$ has essentially $\mathcal{O}\left(2^{J \max _{i \in\{1, \ldots, m\}}\left\{n_{i} / \alpha_{i}\right\}}\right)$ unknowns. However, the complete study of the logarithmic factors (cf. Corollary 4.5) is not an easy task and is highly nontrivial and technical. Moreover, we need to work more on how to transfer the discussion of Section 5 to the sparse $m$-fold tensor product spaces. 


\section{REFERENCES}

[1] G. Allaire and M. Briane. Multiscale convergence and reiterated homogenisation. Proc. Roy. Soc. Edinburgh Sect. A, 126(2):297-342, 1996. MR1386865(97d:35014)

[2] Hans-Joachim Bungartz and Michael Griebel. Sparse grids. Acta Numer., 13:147-269, 2004. MR2249147(2007e:65102)

[3] Nabi Chegini and Rob Stevenson. Adaptive wavelet schemes for parabolic problems: sparse matrices and numerical results. SIAM J. Numer. Anal., 49(1):182-212, 2011. MR2783222

[4] D. Cioranescu, A. Damlamian, and G. Griso. The periodic unfolding method in homogenization. SIAM J. Math. Anal., 40(4):1585-1620, 2008. MR2466168(2009m:35024)

[5] Wolfgang Dahmen. Wavelet and multiscale methods for operator equations. In Acta numerica, 1997, volume 6 of Acta Numer., pages 55-228. Cambridge Univ. Press, Cambridge, 1997. MR,1489256 (98m:65102)

[6] T. Gerstner and M. Griebel. Dimension-adaptive tensor-product quadrature. Computing, 71(1):65-87, 2003. MR2009651 (2004k:65036)

[7] Thomas Gerstner and Michael Griebel. Numerical integration using sparse grids. Numer. Algorithms, 18(3-4):209-232, 1998. MR 1669959 (99m:65042)

[8] M. Griebel and S. Knapek. Optimized general sparse grid approximation spaces for operator equations. Math. Comp., 78(268):2223-2257, 2009. MR2521287 (2010f:65255)

[9] M. Griebel and D. Oeltz. A sparse grid space-time discretization scheme for parabolic problems. Computing, 81(1):1-34, 2007. MR2369419 (2009b:65252)

[10] M. Griebel, P. Oswald, and T. Schiekofer. Sparse grids for boundary integral equations. Numer. Math., 83(2):279-312, 1999. MR1712687 (2000h:65195)

[11] Michael Griebel and Jan Hamaekers. Sparse grids for the Schrödinger equation. M2AN Math. Model. Numer. Anal., 41(2):215-247, 2007. MR2339626(2008h:81042)

[12] Helmut Harbrecht. A finite element method for elliptic problems with stochastic input data. Appl. Numer. Math., 60(3):227-244, 2010. MR2602675(2011c:65261)

[13] Helmut Harbrecht, Reinhold Schneider, and Christoph Schwab. Sparse second moment analysis for elliptic problems in stochastic domains. Numer. Math., 109(3):385-414, 2008. MR 2399150 (2009b:65012)

[14] Viet Ha Hoang and Christoph Schwab. High-dimensional finite elements for elliptic problems with multiple scales. Multiscale Model. Simul., 3(1):168-194, 2004/05. MR2123115 (2005m:65267)

[15] A. Kolmogoroff. Über die beste Annäherung von Funktionen einer gegebenen Funktionenklasse. Ann. of Math. (2), 37(1):107-110, 1936. MR.1503273

[16] C. C. W. Leentvaar and C. W. Oosterlee. On coordinate transformation and grid stretching for sparse grid pricing of basket options. J. Comput. Appl. Math., 222(1):193-209, 2008. MR2462660 (2009j:91102)

[17] Dirk Pflüger, Benjamin Peherstorfer, and Hans-Joachim Bungartz. Spatially adaptive sparse grids for high-dimensional data-driven problems. J. Complexity, 26(5):508-522, 2010. MR2719645 (2011i:65043)

[18] Christoph Reisinger and Gabriel Wittum. Efficient hierarchical approximation of highdimensional option pricing problems. SIAM J. Sci. Comput., 29(1):440-458, 2007. MR2285899(2008b:65107)

[19] Christoph Schwab and Rob Stevenson. Space-time adaptive wavelet methods for parabolic evolution problems. Math. Comp., 78(267):1293-1318, 2009. MR2501051 (2011a:65347)

[20] Christoph Schwab and Radu-Alexandru Todor. Sparse finite elements for elliptic problems with stochastic loading. Numer. Math., 95(4):707-734, 2003. MR2013125 (2004j:65192)

[21] Tobias von Petersdorff and Christoph Schwab. Numerical solution of parabolic equations in high dimensions. M2AN Math. Model. Numer. Anal., 38(1):93-127, 2004. MR2073932 (2005d:65169)

[22] Tobias von Petersdorff and Christoph Schwab. Sparse finite element methods for operator equations with stochastic data. Appl. Math., 51(2):145-180, 2006. MR2212311(2007g:65111)

[23] G. Widmer, R. Hiptmair, and Ch. Schwab. Sparse adaptive finite elements for radiative transfer. J. Comput. Phys., 227(12):6071-6105, 2008. MR2418354 (2010c:85003)

[24] Christoph Zenger. Sparse grids. In Parallel algorithms for partial differential equations (Kiel, 1990), volume 31 of Notes Numer. Fluid Mech., pages 241-251. Vieweg, Braunschweig, 1991. MR.1167882 
Institut für Numerische Simulation, Universität Bonn, Wegelerstr. 6, 53115 Bonn, Germany

E-mail address: griebel@ins.uni-bonn.de

Mathematisches Institut, Universität Basel, Rheinsprung 21, 4051 Basel, SwitzerLAND

E-mail address: helmut.harbrecht@unibas.ch 